\title{
The Relationship between Nutritional Status and Educational Achievements in the Rural School Children of Morocco
}

\author{
El Hioui $\mathrm{M}^{* 1}$, Ahami AOT ${ }^{1}$, Aboussaleh $\mathrm{Y}^{1}$ and Rusinek $\mathrm{S}^{2}$
}

${ }^{1}$ Unit of Clinic and Cognitive Neurosciences and Nutritional Health, Laboratory of Biology and Health, Department of Biology, Faculty of Science, Ibn Tofail University, Kenitra, Morocco

${ }^{2}$ PSITEC Laboratory, University of Lille North of France, Villeneuve d'Ascq, France

${ }^{*}$ Corresponding author: El Hioui M, Unit of Clinic and Cognitive Neuroscience and Nutritional Health, Laboratory of Biology and Health, Department of Biology, Faculty of Science, Ibn Tofail University, Kenitra, Morocco, E-mail: elhioui2000@yahoo.fr

Citation: El Hioui M, Ahami AOT, Aboussaleh Y, Rusinek S (2016) The Relationship between Nutritional Status and Educational Achievements in the Rural School Children of Morocco. J Neurol Neurol Disord 3(1): 101. doi: 10.15744/2454-4981.3.101

\section{Received Date: February 16, 2016 Accepted Date: March 14, 2016 Published Date: March 18, 2016}

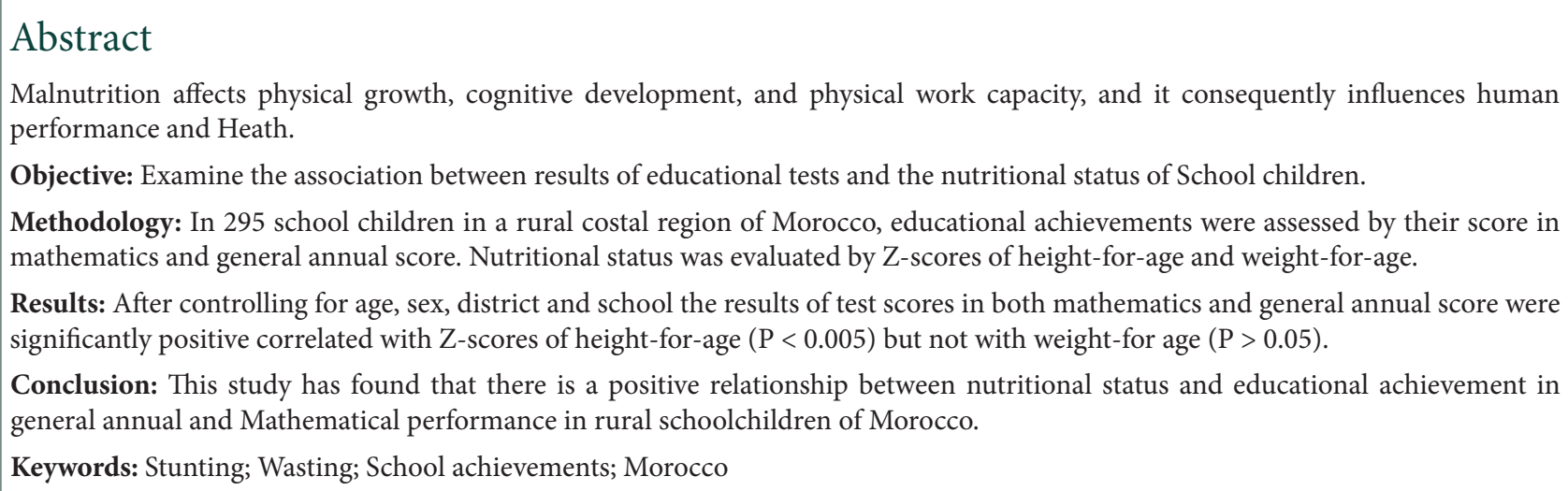

\section{Introduction}

Malnutrition is a serious global issue and each year, some 24 million babies are born too small to lead healthy lives because their mothers were either ill or malnourished [1,2]. Recent reports show that among children under five years of age in developing countries, 206 million are stunted (low height for age) 50 million are wasted (low weight for height) and 167 million are underweight (low weight for age) due to lack of food and the presence of disease, mostly malaria [3].The deficiency in nutritional status of a child is the result of macronutrient and micronutrient deficiencies from inadequate food consumption or from illnesses such as diarrhea [4]. It is quantified using the anthropometric indices of height for age, weight for age, and weight for height; all are expressed as a Z-score, which compare a child's measurements with the measurements of a similar child in a reference, healthy population [5]. A low height for age Z-score (stunting) indicates slow physical growth since the birth, usually due to repeated episodes of poor nutrition and/or episodes of diarrhea and other illnesses. A low weight for height Z-score (wasting) indicates current malnutrition and/or recent episodes of diarrhea and other illnesses, often thought of as a flow measure of malnutrition. A low weight for age Z-score (underweight) reflects both stunting and wasting [6]. In Morocco the last decade has been characterized by a marked improvement in all indicators of the nutritional status of children under 5 years. Among preschoolers, the rate increased from $28.3 \%$ in 1990 to $23 \%$ in 1998 [7]. The return to school is a multifactorial process conditioned by multiple factors which depend on the child (his health, nutrition and its neurocognitive development and the demographic and socioeconomic characteristics. In addition, recent studies show that among the risk factors associated with early cognitive deficits in children, malnutrition in young age, low birth weight and exposure to neurotoxins [8-10].

The objective of this work is to study the relation between the nutritional status, school performance of the children, their cognitive potential and certain socioeconomic and demographic factors of their households in a rural region of Kenitra. 


\section{Materials and Methods}

\section{Study Population}

Two hundred and ninety five children (122 boys and 173 girls) aged6 to 15years were included in this study. The majorityof children live in Ouled Berjal village. All school children present day of the survey were included in the study, except those for which we received a refusal of consent expressed by children or their parents.

The village is not supplied with drinking water and electricity and has septic tanks for sanitation. It is a rural school which is located in the economic region North-western Morocco.

\section{Methods}

Our study is a descriptive cross-sectional survey was conducted using a structured questionnaire.

-Demographic and anthropometric indicators: age, sex, weight, height.

The indicators of malnutrition (height for age and weight for age were determined by Z-scores and calculated with the software Epi info 2000). Stunting and wasting were defined as height-for-age and weight-for-age $Z<-2$ respectively $[11,12]$.

-Socio-economic data: the family's income, the parents' educational status, the number of family members.

\section{Educational achievements}

In total 295 students underwent cognitive tests, the test chosen in our study review and describe writing. The "strong" level for medium (for class exams) and the note or above Mathematics at 6/10, the "average" level for scores below 6/10 but greater than or equal to 4.5 / 10 and "low" for those less than 4.5 / 10.

\section{Statistical analysis}

Chi-2 test and the logistic regression analysis were used to investigate the relationship between the prevalence of malnutrition and the socio-demographic factors and school achievement. Differences were considered statistically significant at a p $<0.05$.

\section{Results}

Table 1 presents the descriptive results of the anthropometric characteristics such as the height for age (HAZ) and weight for age (WAZ) and body mass index (BMI) in all classes (grades 4-8), from since the age of 6 to 16 years. The average size for Age -0.56 \pm 1.4 , weight for age $0.25 \pm 1.04$.

\begin{tabular}{|c|c|}
\hline Parameters & Mean \pm standard deviation \\
\hline Weight $\mathrm{Kg}$ & $29,08 \pm 9,06$ \\
\hline Height in cm & $134,6 \pm 113,9$ \\
\hline Height for age (mean Z score) & $-0,56 \pm 1,4$ \\
\hline Weight for age (mean Z score) & $-0,25 \pm 1,04$ \\
\hline
\end{tabular}

Table 1: Overall results of anthropometric parameters

Table 2 shows the anthropometric status of children by sex and age. These results show that the level of stunting and wasting among boys is $12.2 \%, 12.3 \%$, respectively.

\begin{tabular}{|c|c|c|c|c|c|c|}
\hline & \multicolumn{3}{|c|}{ Stunting } & \multicolumn{3}{|c|}{ Wasting } \\
\hline & $<-2 \mathrm{Z}$ & $\geq-2 \mathrm{Z}$ & $\mathbf{P}$ & $<-2 \mathrm{Z}$ & $\geq-2 \mathrm{Z}$ & $\mathbf{P}$ \\
\hline \multicolumn{7}{|c|}{ Sex } \\
\hline Girls & $10(8,2 \%)$ & $112(91.8 \%)$ & \multirow{2}{*}{$\mathrm{P}>0.05$} & $16(13.1 \%)$ & $106(86.9 \%)$ & \multirow{2}{*}{$\mathrm{P}>0.05$} \\
\hline Boys & $16(12.2 \%)$ & $115(87.8 \%)$ & & $21(12.3 \%)$ & $150(87.7 \%)$ & \\
\hline \multicolumn{7}{|c|}{ Age (years) } \\
\hline$\leq 10$ & $7(4.4 \%)$ & $152(94.6 \%)$ & \multirow{2}{*}{0,0034} & $13(8.2 \%)$ & $146(91.8 \%)$ & \multirow{2}{*}{0,012} \\
\hline$>10$ & $19(14.2 \%)$ & $115(85.8 \%)$ & & $24(17.9 \%)$ & $110(82.1 \%)$ & \\
\hline
\end{tabular}

Table 2: Anthropometric status of children by sex and age

Table 3 shows the distribution of children according to their academic performance (average math scores or the average) by sex. We note respectively $30.1 \%$ of girls and $38.7 \%$ of small boys in mathematics and $17.1 \%$ of girls and $37.3 \%$ of small boys to the general average. 


\begin{tabular}{|c|c|c|c|c|c|c|c|c|}
\hline \multirow{2}{*}{} & \multicolumn{4}{|c|}{ Girls } & \multicolumn{4}{c|}{ Boys } \\
\cline { 2 - 9 } & \multicolumn{2}{|c|}{ Math } & \multicolumn{2}{c|}{ Annual average } & \multicolumn{2}{c|}{ Math } & Annual average \\
\cline { 2 - 9 } & N & $\%$ & N & $\%$ & N & $\%$ & N & $\%$ \\
\hline Low & 37 & 30.1 & 20 & 17.1 & 65 & 38,7 & 60 & 37,3 \\
\hline Average & 57 & 46,3 & 61 & 52.1 & 60 & 35,7 & 60 & 37,3 \\
\hline Forte & 29 & 23,6 & 36 & 30.8 & 43 & 25,6 & 41 & 25,4 \\
\hline
\end{tabular}

Table 3: Frequency of school performance (reliability, medium, high) for Mathematics and the annual average student

Table 4 shows that the height for age was positively correlated $(\mathrm{p}=0.009)$ with the annual ranking of the student and the average math $(\mathrm{p}=0.007)$. No significant difference was found between weight for age and academic performance.

\begin{tabular}{|c|c|c|}
\hline & IC (95\%) & P \\
\hline Math & & \\
- HAZ (Height for age) & $-0.24-0.19$ & 0,007 \\
- WAZ (weight for age) & $-0.057-0.25$ & 0.43 \\
\hline Annual average & & \\
- WAZ & $0.08-0.12$ & 0,009 \\
- WAZ & $-0.043-0.25$ & 0.6 \\
\hline
\end{tabular}

Table 4: Linear regression between the average and the Math Average and physical indicators

\section{Discussion}

This study is conducted in a rural area of Kenitra. The study of population is consisted of $122(41.6 \%)$ girls and 173 (58.4\%) boys. The average age of our study population is $10.02 \pm 2.5$ years with a range of 6 to 16 years.

The problem of stunting young child starts early at the age of 3 years and reflects early malnutrition. Food diversification and environmental health are the determining factors during this period. But the general constraints of rural areas are debilitating as physical to food that is not regular. In addition, access to clean water is recently in Morocco. Besides the education of women and their social status are key factors for health of child especially when economic conditions are difficult. Malnutrition was more prevalent in children with illiterate fathers compared to other children. The prevalence of wasting and stunting was lower in children from economically better off families. The results of the regression analysis show that the prevalence of stunting was $12.2 \%$ and the prevalence of wasting $12.3 \%$ among boys. Also, the malnutrition was significantly more frequent among boys than girls. This increase has also been reported by Baba, et al. in a study in Lebanon [13]. This confirms the results of the literature reporting that stunting tends to increase with age for children. Possible explanations are the lack of adequate complementary infant feeding both quantitatively and qualitatively. It also appears that the transition to family meals is not always beneficial to the child and that food diversification is associated with the growth of children [14]. We note respectively $30.1 \%$ of girls and $38.7 \%$ of boys rated as poor for math and $17.1 \%$ of girls and $37.3 \%$ of boys rated as poor to average, so boys appear less successful than girls. So the boys appear less efficient than the girls, contrary to the study of Doyle, et al. reveals that girls have more learning difficulties in mathematics than boys [15].

The effect of nutritional status on the academic performance of the student was not confirmed in our study, as a causal effect by multiple regression analysis. This is consistent with what is observed in the literature since, also noted that there is no significant relationship between academic achievement of children and their nutritional status [14]. Grantham-McGregor, et al. by cons reviewed the literature and found that early stunting ( $\mathrm{HAZ}<-2 \mathrm{SD}$ from median) and poverty in the first five years of life were associated with lower subsequent cognitive abilities, school achievement, and productivity in adult life [16]. This can be explained in this case by the fact that the majority of children surveyed are not suffering from advanced malnutrition. Indeed, recent decades have seen a remarkable improvement in nutritional indicators among young Moroccan children.

The 1990 survey on the living standards of Moroccan households reported a rate of $23.9 \%$ of height deficit (Z-score of height for age $<-2.0$ ) in children under 11 years (Directorate statistics, 1992). Among preschoolers, the rate increased from $28.3 \%$ in 1990 to $23 \%$ in 1998 [7]. In our case, stunting and wasting among boys is $12.2 \%, 12.3 \%$ respectively of schoolchildren. Moreover Galal and Hulett found in their literature review that nutritional status is rarely considered as a causal factor and priority of academic performance but generally as an underlying factor [17]. They found that by cons are the demographic and socioeconomic characteristics that are most frequently cited as important determinants of attendance and academic performance of children. In particular, they stressed like other authors that parents encourage the return of their children if they have a high level of education [18]. A different study has similarly reported that in urban or rural areas where external influences a children's mental performance are available; there may be no significant difference in cognitive performance between well-nourished children and those showing signs of wasting [19]. 


\section{Conclusion}

The results of the present study show that school performance is a multifactorial process determined by multiple factors, including those dependent on child and his family.

\section{Acknowledgment}

The authors are very grateful to the children, their parents, and the staff of the OueladBerjal High School and to the staff of OueladBerjal Hospital for their considerable help and contributions to this research.

\section{References}

1. UNICEF (1998) The state of the world's children New York: Oxford University Press.

2. Fernandez ID, Himes JH, de Onis M (2002) Prevalence of nutritional wasting in populations: building explanatory models using secondary data. Bull World Health Organ 80: 282-91.

3. Pelletier DL (1994) The potentiating effect of malnutrition on child mortality: epidemiologic evidence and policy implications. Nutrition Reviews 52: 409-15.

4. Behrman JR, Alderman H, Hoddinott J (2004) Hunger and Malnutrition. In Lomborg B, 2nd ed, Global Crisis, Global Solutions, Cambridge University Press, Cambridge.

5. Martorell R (1985) Child Growth Retardation: A Discussion of Its Causes and Its Relationship to Health. In Blaxter K Waterlow JC, Eds, Nutritional Adaptation in Man, John Libby, London.

6. Wisniewski SLW (2010) Child Nutrition, Health Problems, and School Achievement in Sri Lanka. World Dev 38: 315-32.

7. Benjelloun S (2002) Nutrition transition in Morocco. Center for Disease Control and Prevention 2001, National Center for Health Statistics-Clinical Growth Charts. Public Health Nutr 5: 135-140.

8. Galler JR, Ramsey F, Solimano G, Lowell WE (1983) The Influence of Early Malnutrition on Subsequent Behavioral Development. II. Classroom Behavior. J Am Acad Child Psychiatry 24: 16-22.

9. Astbury J, Orgili AA, Bajuk B, Yu VY (1985) Neonatal and Neuro developmental Significance of Behavior in Very Low Birthweight Children. Early Hum Dev 11: $113-21$.

10. Needleman HL, Bellinger DC (1981) The Epidemiology of Low-Level Lead Exposure in Childhood. J Child Psychiatry 20: 496-512.

11. Cogill B (2003) Anthropometric Indicators Measurement Guide, Food and Nutrition Technical Assistance Project, Academy for Educational Development, Washington DC 2003.

12. United Nations Children's Fund (1986) How to Weigh and Measure Children: Assessing the Nutritional Status of Young Children in Household Surveys. United Nations Department of Technical Co-operation for Development and Statistical Office (UNICEF), New York.

13. Baba N, Khuzama S, El-Sheikh Ismail L, Adra N (1996) Comparison of nutritional status of pre-school children at day care centres and at home from different socioeconomic backgrounds in Beirut. J Human Nutri Diet 9: 8-103.

14. El Hioui M, Azzaoui FZ, Ahami AOT, Aboussaleh Y (2011) Nutritional Status and School Achievements in a Rural Area of Anti-Atlas, Morocco. Food Nutri Sci 2: 878-83.

15. Doyle AE, Faraone SV, DuPre EP (2001) Separating attention deficit hyperactivity disorder and learning disabilities in girls: a familial risk analysis. Am J Psychiatry 158: 1666-72.

16. Grantham-McGregor S, Cheung YB, Cueto S, Glewwe P, Richter L (2007) The International Child Development Steering Group Developmental potential in the first 5 years for children in developing countries. Lancet 369: 60-70.

17 .Galal O, Hulett J (2003) The relationship between nutrition and children's educational performance: a focus on the United Arab Emirates. British Nutrition Foundation. Nutri Bullet 28: 11-20.

18. Hack M, Breslau N, Aram D, Weissman B, Klein N, et al. (1992) The Effect of Very Low Birth Weight and Social Risk on Neurocognitive Abilities at School Age. J Dev Behav Pediatr 13: 412-20.

19. Cotton J (1982) Evaluation Research on the PL 480: School Feeding Program in Haiti. Port-au-Prince, Haiti: USAID.

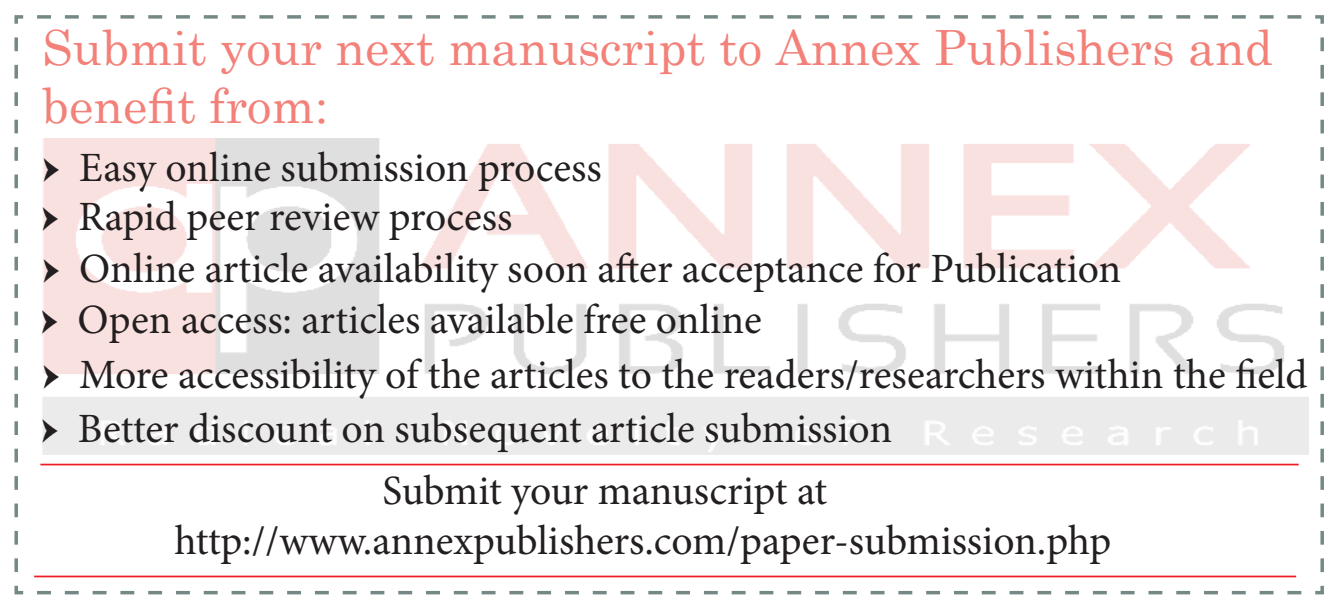

